Supporting Information

\title{
Incorporation of Lithium Fluoride Restraining Thermal Degradation and Photodegradation of Organometal Halide Perovskite Solar Cells
}

Alan Jiwan Yun, ${ }^{+}$Jinhyun Kim, ${ }^{+}$Bumjin Gil, Hyungsub Woo, Kimin Park, Jaemin Cho, and Byungwoo Park*

Department of Materials Science and Engineering, Research Institute of Advanced Materials, Seoul National University, Seoul 08826, Korea

\footnotetext{
*E-mail: byungwoo@ snu.ac.kr; Phone: +82-2-880-8319; Fax: +82-2-885-9671.

+ These authors contributed equally to this work.
} 

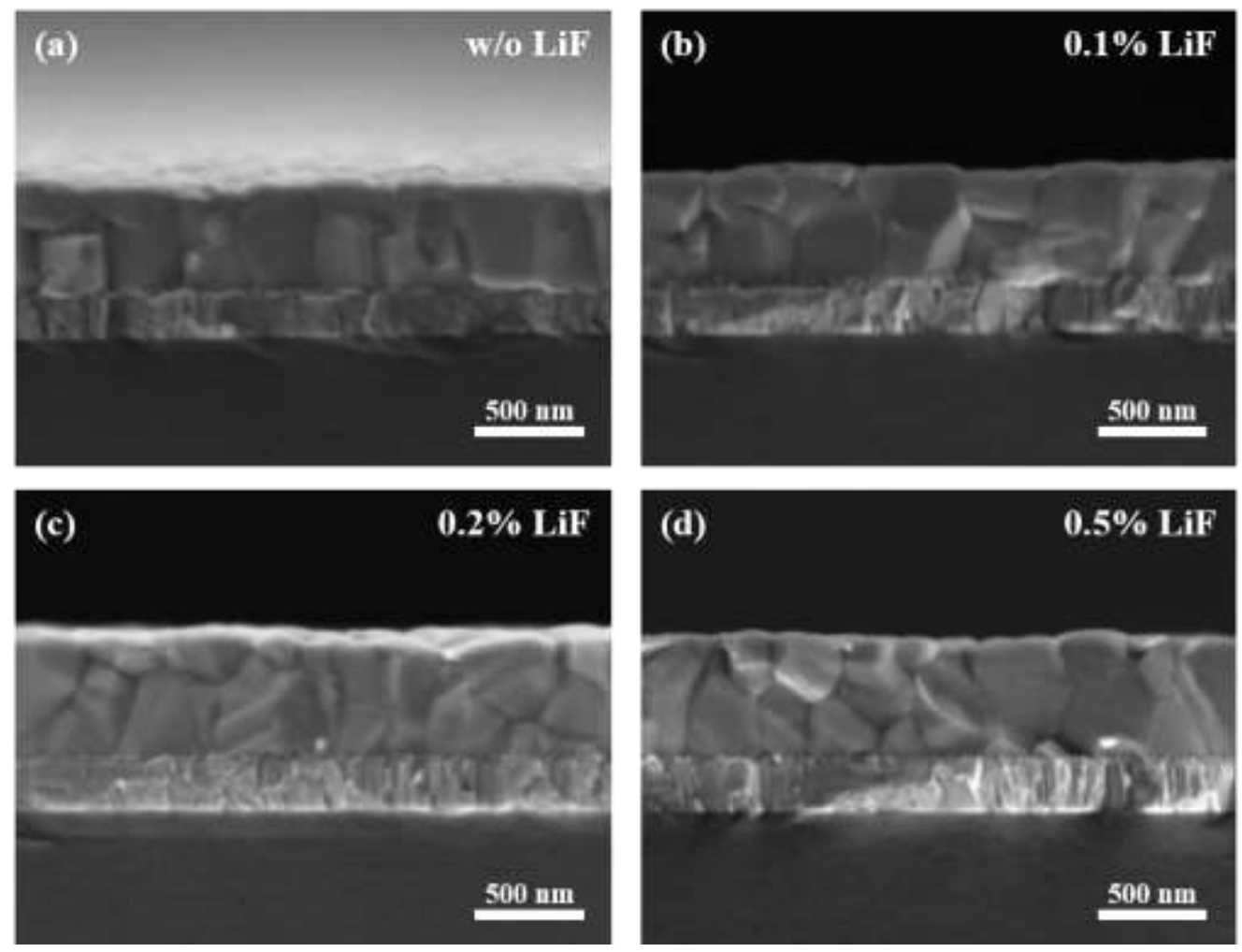

Figure S1. Cross-sectional SEM images of CsFAMA:LiF films. Perovskite films fabricated on the ITO/SnO 2 substrates: (a) without $\mathrm{LiF}$, and (b-d) with $0.1,0.2$, and $0.5 \mathrm{~mol} . \% \mathrm{LiF}$, respectively. 

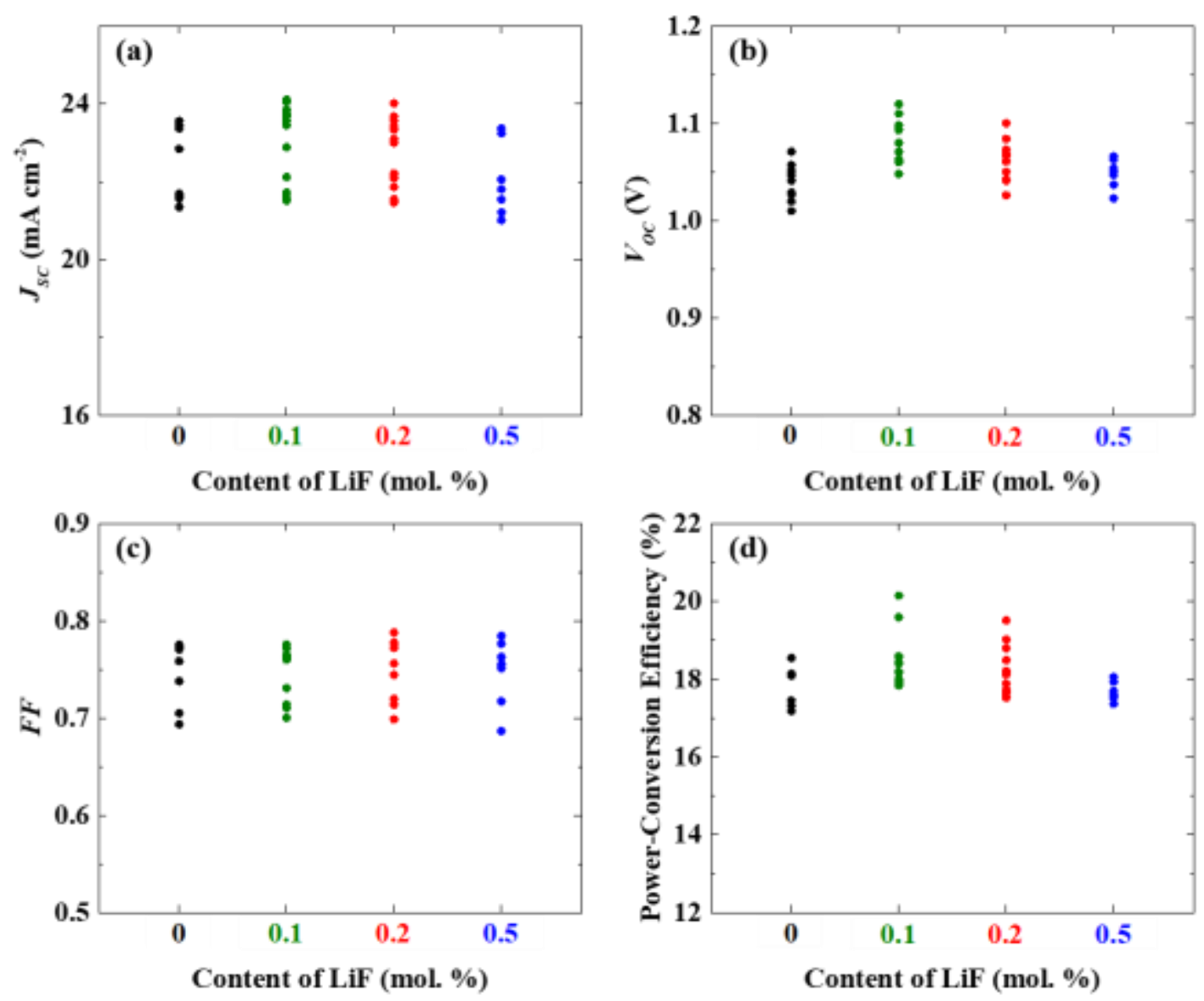

Figure S2. Photovoltaic performance of LiF-incorporated perovskite solar cells (PSCsLiF). (a) Short-circuit currents $\left(J_{S C}\right)$, (b) open-circuit voltages $\left(V_{O C}\right)$, (c) fill factors $(F F)$, and (d) power-conversion efficiencies of the devices. 


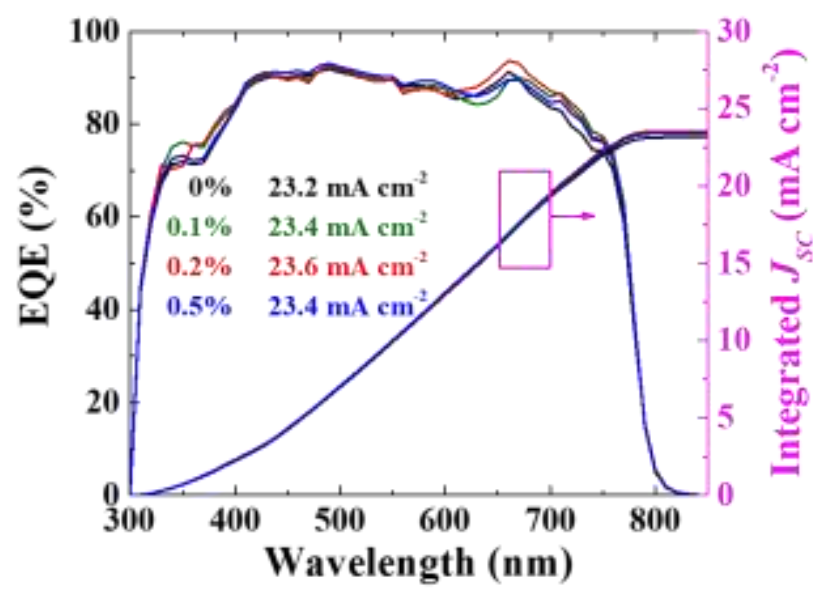

Figure S3. Incident photon-to-electron conversion efficiency (IPCE) measurement for the PSCs-LiF. External quantum efficiency (EQE) spectra and integrated short-circuit current densities $\left(J_{S C}\right)$ of the devices. 

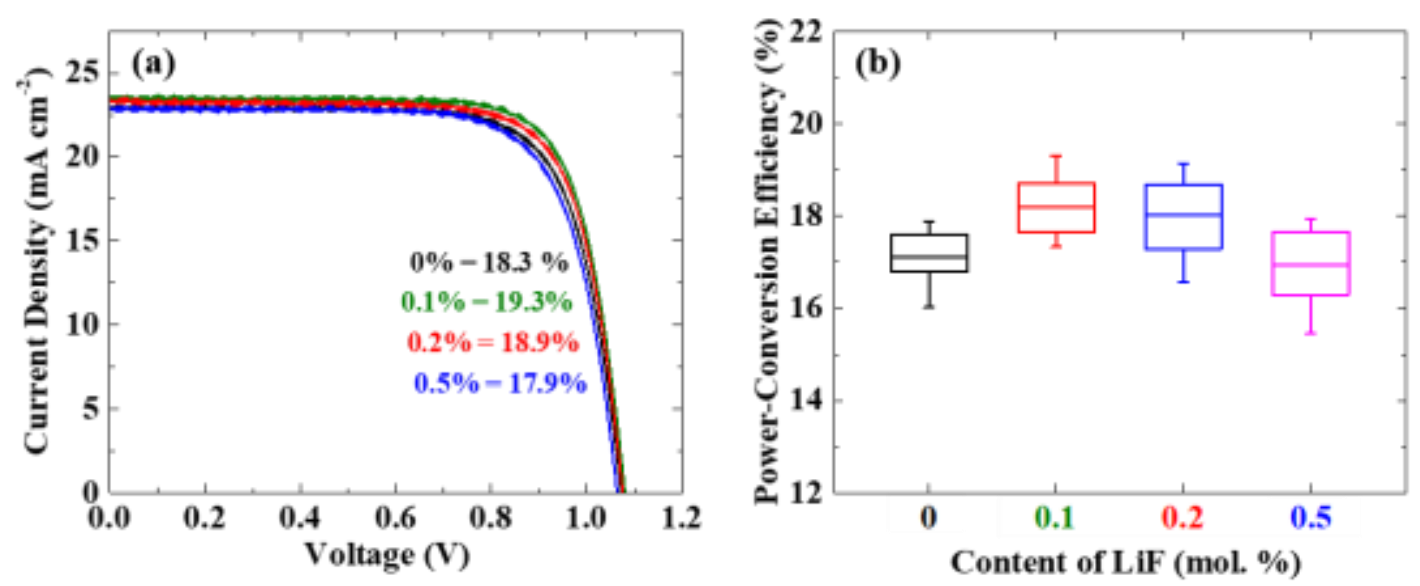

Figure S4. Photovoltaic performance of PSCs-LiF cells with PTAA HTL. (a) $J$ - $V$ curves of the champion cells with PTAA HTL. (b) The initial PCEs of the PTAA-based devices used for the stability tests. 

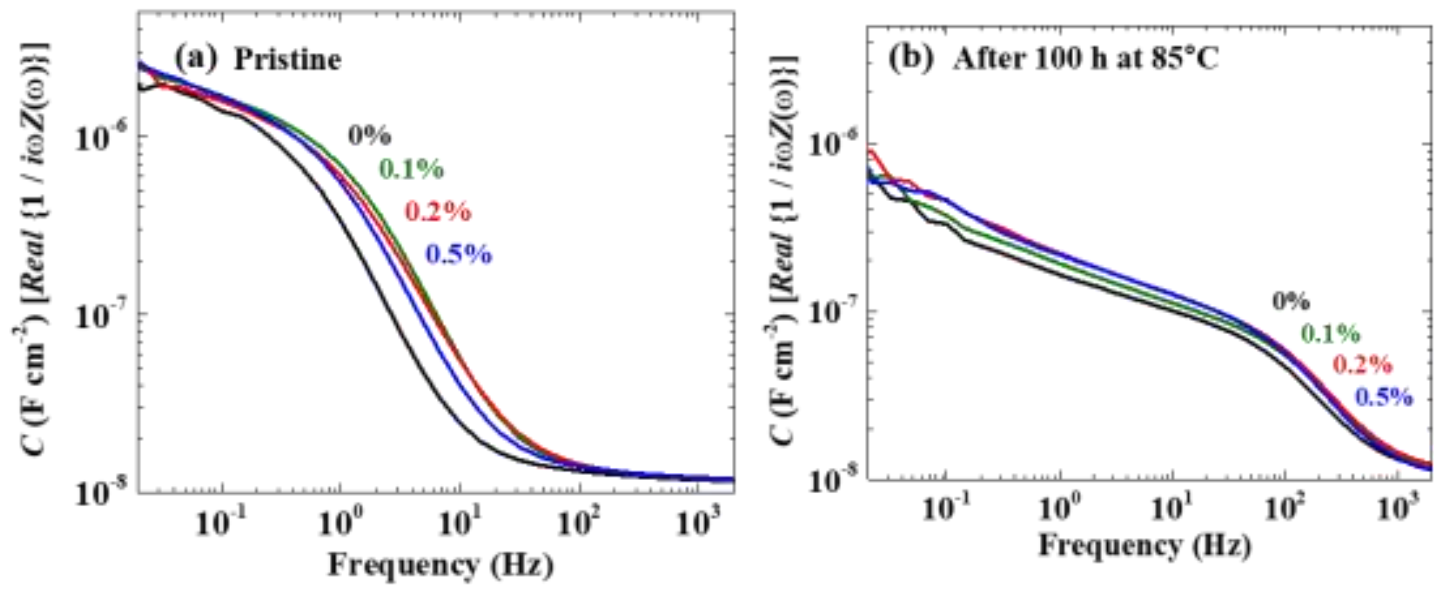

Figure S5. Capacitance analysis of the PSC-LiF. Capacitance vs. frequency plots of devices, (a) before and (b) after the thermal stability test (Figure 3). 


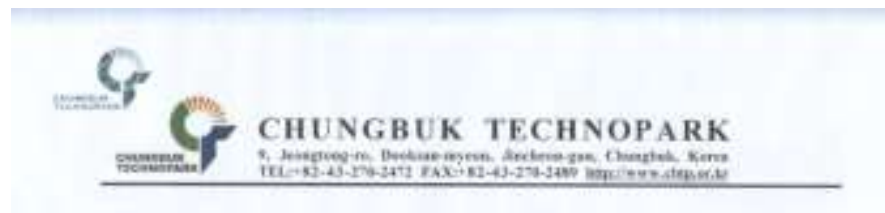

CTet tien at lisats

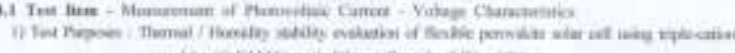

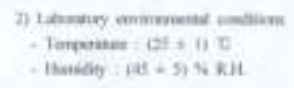

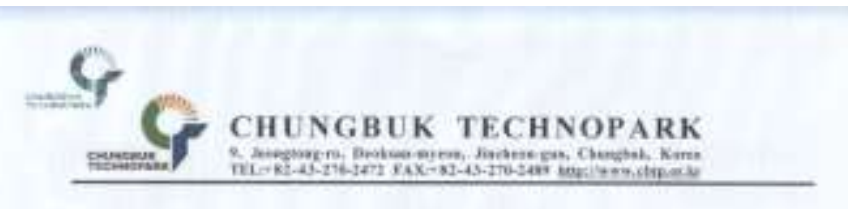

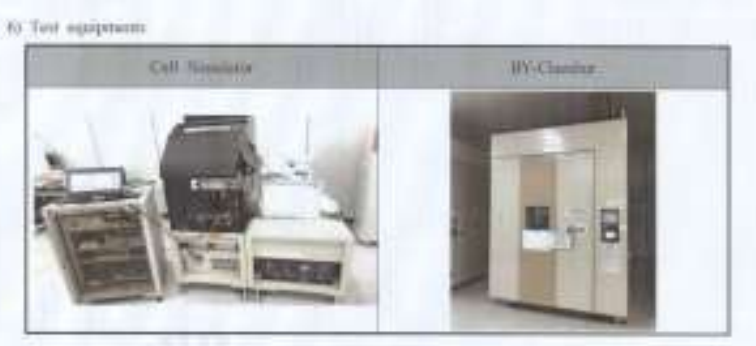

4.2 Yeat Henat

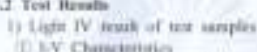

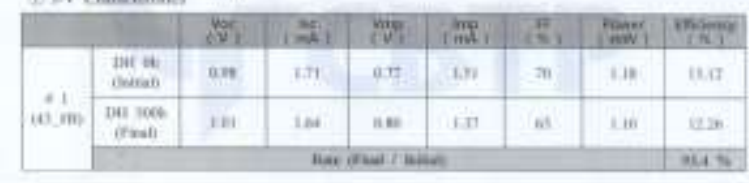

ats cane
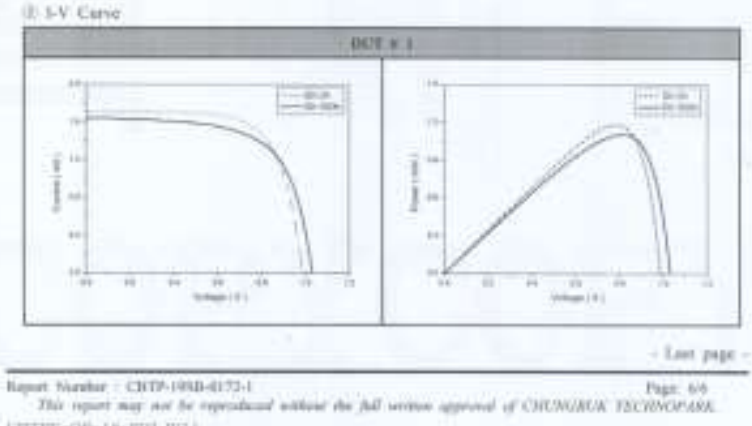

nime

Figure S6. Certified thermal stability of flexible PSC-LiF. The certificate of the flexible PSC-LiF before and after thermal/humidity stability test employing the ISOS standards (Figure 5). Logo courtesy of Chungbuk Technopark. 